\title{
Thickness of adherent mucus gel on colonic mucosa in humans and its relevance to colitis
}

\author{
R D Pullan, G A O Thomas, M Rhodes, R G Newcombe, G T Williams, A Allen, J Rhodes
}

\begin{abstract}
The thickness of adherent mucus gel on the surface of colonic mucosa was measured in surgically resected specimens from 46 'control' patients most of whom had carcinoma of the colon; 12 were from right colon, 17 left colon, and 21 from rectum. In addition specimens were examined from 17 patients with ulcerative colitis and 15 patients with Crohn's disease. In controls a continuous layer of mucus was readily seen on specially prepared sections viewed by phase contrast illumination. Mean values for right and left colon and rectum were 107 (48), 134 (68), and 155 (54) $\mu \mathrm{m}$ respectively with a significant difference between right colon and rectum $(p=0.015)$. Values in ulcerative colitis showed greater variation and in those areas with acute inflammation mucosa was denuded of the mucus layer. In contrast, values for Crohn's disease were normal or greater than normal in thickness - right colon 190 (83) $\mu \mathrm{m}$ compared with $10748 \mu \mathrm{m}$, $p=0.0093$. A series of validation experiments are described for the method used to measure mucus thickness. The possible role of mucus in the pathogenesis of inflammatory bowel disease is discussed.

(Gut 1994; 35: 353-359)
\end{abstract}

Mucus in the gastrointestinal tract adheres as a gel to surface epithelium producing an interface

Department of Gastroenterology, University Hospital of

Wales, Cardiff R D Pullan

G A O Thomas

J Rhodes

Departments of Medical Computing and Statistics R G Newcombe and Histopathology, University of Wales
College of Medicine, Cardiff

G T Williams

Departments of Surgery $M$ Rhodes

and Physiological Sciences, The Medical School, Newcastle upon
Schen Tyne

A Allen

Correspondence to: Professor J Rhodes, Department of Gastroenterology, University Hospital of Wales, Heath Park, Cardiff CF4 4XW.

Accepted for publication 13 July 1993 between the lumen and mucosa. ${ }^{1-4}$ In the stomach a continuous layer of mucus forms a barrier between erosive gastric contents and the underlying mucosa..$^{5-7}$ Factors responsible for maintaining this mucus bicarbonate barrier have given insight into mechanisms responsible for peptic ulcer and gastric mucosal damage. ${ }^{348}$ The situation in the colon is somewhat analogous but few findings have been made on colonic mucus gel to determine its relevance in disease. In ulcerative colitis mucus is both quantitatively and qualitatively different from controls, ${ }^{9-13}$ and the differences may influence physical properties of the gel and its role as a putative protective barrier. ${ }^{14}$ We have examined the thickness of the adherent layer of colonic mucus in humans using fresh surgical specimens and have looked parbowel disease.

\section{Methods}

PATIENTS

Specimens of colon were obtained as a consequence of surgical resection from three groups ticularly at changes in patients with inflammatory of patients - 'controls', ulcerative colitis, and Crohn's disease.

The 'control' group consisted of 46 patients who did not have inflammatory bowel disease; 25 were male and 21 female with a mean age of 66 years (range 23 to 90 ). Forty two had carcinoma, two diverticular disease, one an appendix mass, and one a submucosal lipoma. Specimens included 12 from the right colon, 17 left colon, and 21 from the rectum.

Seventeen patients had ulcerative colitis, 10 males and seven females with a mean age of 47 years (range 22 to 79). Fourteen had a proctocolectomy as an elective procedure for persistent severe disease, one a colectomy and ileorectal anastomosis, and two specimens were for completion proctectomy alone. Fifteen patients had Crohn's disease, 11 of whom were female, with a mean age of 38 years, range 18 to 64 . Ten had ileocaecal resections for ileocaecal disease, two had left colectomies, one had a proctectomy, and two had total colectomies. Details of drug treatment, which included steroids, 5-amino salicyclic acid (5-ASA) and azathioprine taken immediately before surgery was recorded.

The diagnostic categories for inflammatory bowel disease were based on criteria defined by Lennard-Jones ${ }^{15}$ and included full pathological data. All were elective operations and entailed preoperative bowel preparation with either Picolax (Ferring, UK) or Kleanprep (Nordica, UK).

\section{PREPARATION OF MUCOSAL SECTIONS}

Resected specimens were examined within 20 minutes of surgical removal and samples of mucosa dissected from them to prepare sections for measurement of surface mucus thickness. Cases were selected on the basis that specimens were available within 20 minutes of resection. They were opened carefully along the antimesenteric border and any surface contamination gently washed away with drops of physiological saline from a $20 \mathrm{ml}$ syringe. In the control group a standard protocol was used to take samples one centimetre square, dissected from 'normal' mucosa at least $5 \mathrm{~cm}$ from any abnormality such as a tumour and away from the resection margin. Dissection of the mucosa from the muscularis mucosa was an essential step in the preparation of samples before cutting sections for measurement of mucus thickness; the technique we used does not disturb the mucus layer. In the right colon most lesions were carcinoma of the caecum and samples came from mid-ascending colon. In specimens from inflammatory bowel disease several areas were sampled from around the colon to include both inflamed and comparatively normal mucosa. For both controls and colitis an 
attempt was made to take at least two samples from each area of colon examined. Specimens were further classified anatomically - with right colon defined as caecum to mid-transverse colon and left colon as mid-transverse to distal sigmoid.

Each sample was placed without distortion on filter paper (ashless; grade 41, Whatman, UK) and the surface was kept moist with drops of saline. Sections were cut with a pair of parallel razor blades mounted between two perspex blocks and separated by $1.6 \mathrm{~mm} .^{7}$ This was done by pressing vertically on the mucosal sample to ensure it was cut at right angles. Each section was teased from the blades by gentle traction on the filter paper and placed on its side on a slide, taking care to ensure the mucosal surface remained at right angles to the slide. The whole procedure took less than five minutes. The macroscopic appearance of specimens was noted. The same sections were later preserved in formal saline acetic acid solution ( $10 \%$ formalin, $2 \%$ acetic acid in $0.9 \% \mathrm{NaCl}$ ).

Saline

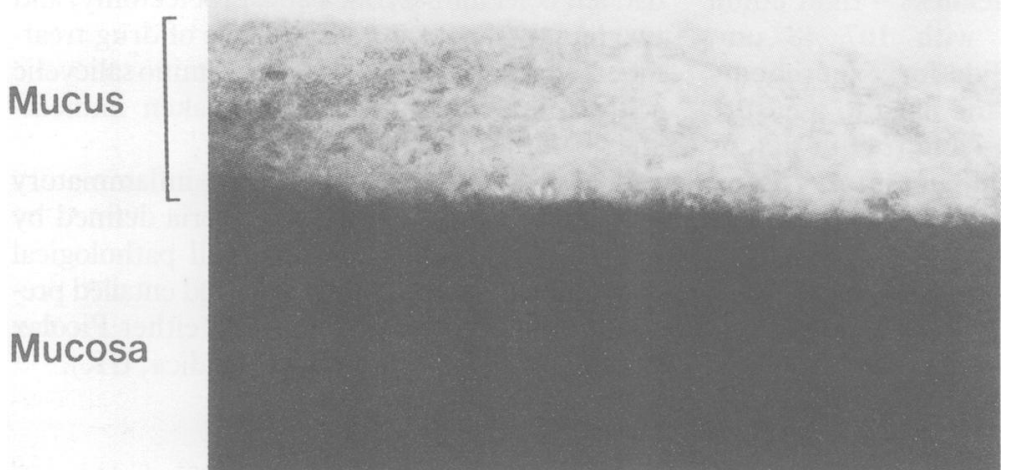

Figure 1: Photomicrograph of the adherent mucus layer on the surface of a thick section of fresh colonic mucosa under phase contrast illumination (100× magnification). Limits of the mucus layer are the two interfaces saline-mucus (upper) and mucus-mucosa (lower). The thickness shown (bar) is $150 \mu \mathrm{m}$.
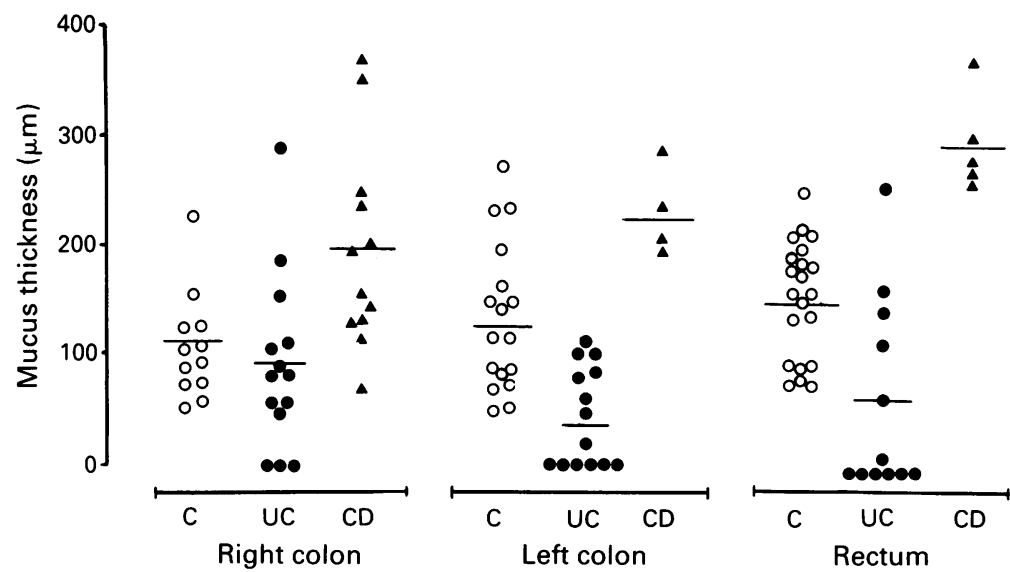

Figure 2: Thickness of adherent colonic mucus $(\mu m)$ in controls $(C) \bigcirc$, ulcerative colitis (UC) and Crohn's disease (CD) $\triangle$. Control specimens (50) were obtained from 46 patients most of whom had carcinoma. Forty UC specimens were from 17 patients and 21 Crohn's disease specimens from 15 patients. The number of measurements from each resected specimen was determined by the extent of the resection; many with inflammatory bowel disease with a proctocolectomy had samples from each of the three sites. Means are shown.

\section{MEASUREMENT OF MUCUS THICKNESS}

Thickness of surface mucus on each section was measured with an inverse microscope $(\times 100$ or $\times 200$ magnification) using phase contrast illumination and focused on the visible mucus layer (Fig 1). Measurements were made at $500 \mu \mathrm{m}$ intervals with a calibrated eyepiece graticule; a minimum of 10 readings were made on each section with at least two samples from each specimen.

For each 'control' specimen an overall mean was taken from the readings obtained on all the sections; each 'control' specimen was represented by a single data point. For specimens of ulcerative colitis and Crohn's disease several areas were defined on the specimen to include the anatomical sites right colon, left colon, and rectum as well as both inflamed and comparatively uninflamed areas where this was possible (Fig 2). From each chosen site a single sample was taken and sections prepared to record mucus thickness; the mean of at least 10 measurements was taken. Each specimen was represented by several data points based on anatomical site.

\section{HISTOLOGICAL EXAMINATION}

The dissected sections from patients with inflammatory bowel disease were subsequently fixed in formal saline acetic acid. Histological sections were then prepared, stained with haematoxylin and eosin, and used to quantitate the severity of epithelial damage and inflammatory activity using criteria based on Truelove and Richards. ${ }^{16}$ Grade 0 referred to absence of polymorphs, 1 to small numbers of polymorphs in the lamina propria with minimal infiltration of crypts, 2 described prominent polymorphs in the lamina propria with infiltration $>50 \%$ crypts, 3 referred to a florid polymorph infiltrate with crypt abscesses, and 4 to florid acute inflammation with ulceration. The surface epithelium was maintained in grades 1 to 3 .

\section{VALIDATION OF THE METHOD FOR THE} MEASUREMENT OF MUCUS THICKNESS

To validate methodology for the measurement of mucus thickness, a series of tests were done.

(1) The interface between surface gel and overlying saline solution was identified by Indian ink in the bathing solution. The particles of ink failed to penetrate the mucus layer but concentrated at the saline-gel interface.

(2) The effect of forceful washing with saline on the mucus gel was examined in five samples. Adjacent samples were taken - one prepared in the conventional manner and the other first washed with saline; washed samples lost their mucus layer.

(3) Fresh, unfixed sections were treated with periodic acid - Schiff reagent (PAS), which readily stained the surface layer in a way consistent with mucus (Fig 3 top). The usual procedures were modified - vigorous washing was avoided and samples were simply dipped in each reagent.

(4) Sections were specially prepared to make it possible to stain the layer of mucus gel. Because the mucus layer is labile and lost by dehydration 

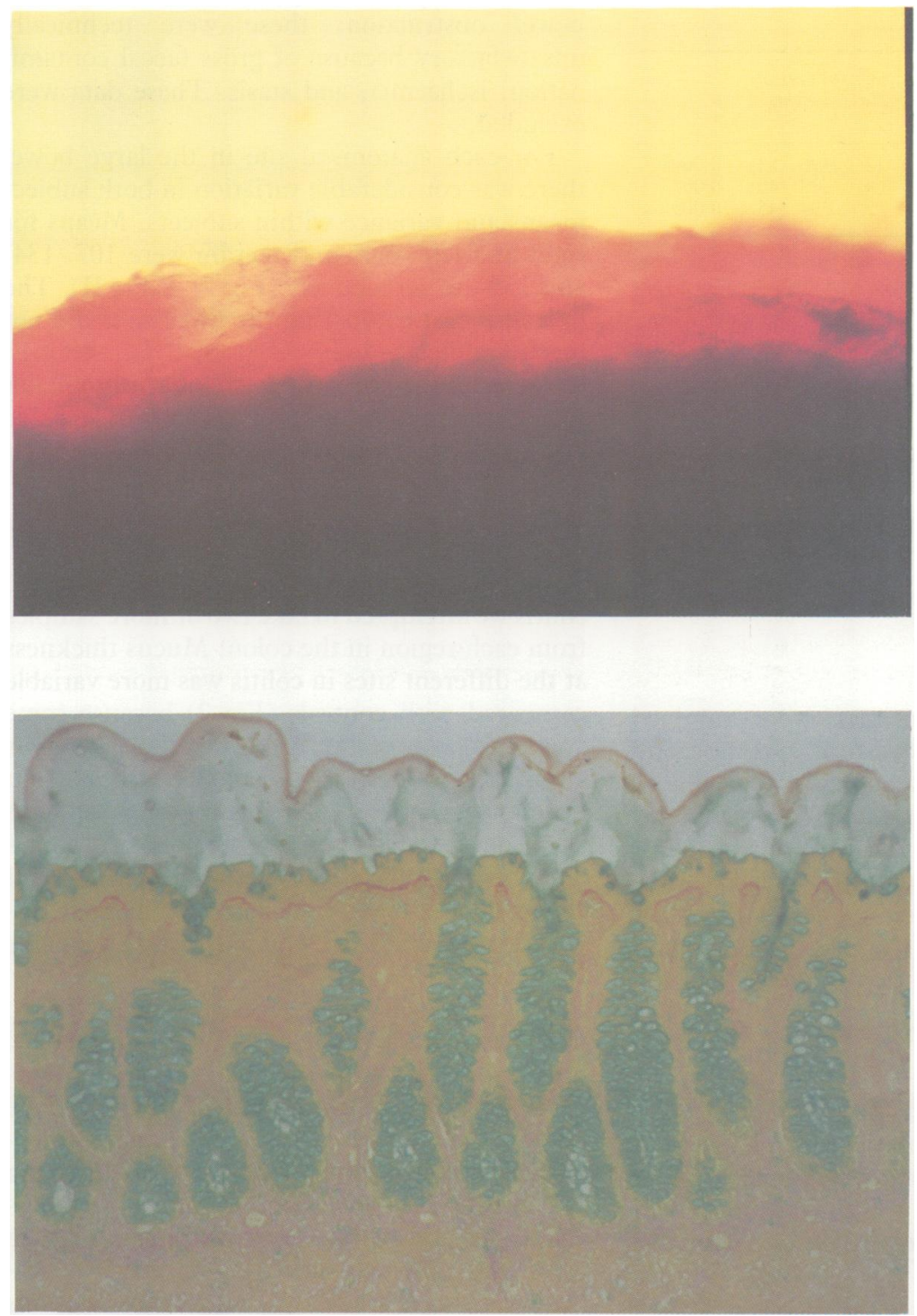

Figure 3: (top) Fresh, unfixed thick mucosal section stained with periodic acid-Schiff-reagent and viewed on inverted microscope at $\times 100$; (bottom) Mucosal sample stained histochemically with alcian blue and van Gieson counterstain after stabilising the mucus gel layer by cryostat and molten agar. $\mu \mathrm{m}$ in thickness stained with haematoxylin and eosin, PAS with diastase, and Alcian blue (Fig 3 bottom). The surface mucus was moderately well preserved by this technique and gave positive indications with each of the histochemical stains (Fig 3 bottom).

(5) Stability of the mucus layer over one hour was examined by repeated measurements from the same section after 30 and 60 minutes. This was done in 12 specimens and gave consistent values over this period (Table I).

(6) The effect of the thickness of mucosal sections was varied to examine the effect of this on measurements of the mucus layer. Sections of $1.0,1 \cdot 6$, and $2.6 \mathrm{~mm}$ thickness were taken from the same piece of colonic mucosa and mucus thickness recorded in 11 specimens. Measurements showed no significant difference (Table I).

(7) Variation in measurements of mucus thickness between adjacent sections of mucosa was examined by cutting three sections simultaneously, using four razor blades with three spacers in the cutter. Ten specimens examined in this way showed no significant difference (Table I).

(8) Mucus thickness was also measured from both sides of the section after turning it through $180^{\circ}$ longitudinally; this was done with 10 specimens and the paired findings were very similar (Table I).

(9) Two independent observers made measurements separately to estimate interobserver variability in 20 samples of mucosa from control patients. There was a good correlation $(r=0.921)$, with a mean (SD) of the paired differences being $5 \cdot 8(24 \cdot 4)$; this shows no significant difference between observers $(\mathrm{t}=1.03 ; \mathrm{p}=0.31)$.

(10) The effect of different bowel preparations, which were those preferred by the surgeon, was examined in the control group. Twenty six had been given Picolax and 24 Kleanprep; comparison of the thickness of mucus in the two groups gave no significant difference (means 139 $\mu \mathrm{m}$ and $128 \mu \mathrm{m}$ respectively; $\mathrm{t}=1 \cdot 0 ; \mathrm{p}=0 \cdot 32$ ).

during tissue handling with fixation special techniques are required to preserve it. Fresh sections were snap frozen with liquid nitrogen and isopentane, allowed to dry overnight at $-40^{\circ} \mathrm{C}$, and then dipped in molten agar $0.5 \%$ (Agar powder, BDH Chemicals Ltd) and cooled until the agar set. Tissue was fixed in Carnoy's reagent $(60 \%$ absolute alcohol, $30 \%$ chloroform, $10 \%$ glacial acetic acid, vol/vol) and sections 10

TABLE I Validation of the treatment of mucus thickness

\begin{tabular}{|c|c|c|c|c|c|}
\hline \multicolumn{2}{|c|}{ Experiment } & \multicolumn{4}{|l|}{$\begin{array}{l}\text { No of } \\
\text { specimens }\end{array}$} \\
\hline 1 & Effect of time & 12 & $\begin{array}{l}0 \min \\
160(47)\end{array}$ & $\begin{array}{l}30 \min \\
158(52)\end{array}$ & $\begin{array}{l}60 \min \\
161(48)\end{array}$ \\
\hline 2 & Effect of section thickness & 11 & $\begin{array}{l}1.0 \mathrm{~mm} \\
227(32)\end{array}$ & $\begin{array}{l}1.6 \mathrm{~mm} \\
226(35)\end{array}$ & $\begin{array}{l}2.6 \mathrm{~mm} \\
229(39)\end{array}$ \\
\hline 3 & Variation in adjacent sections & 10 & $\begin{array}{l}1 \\
234(31)\end{array}$ & $\begin{array}{l}2 \\
239(34)\end{array}$ & $\begin{array}{l}3 \\
236(32)\end{array}$ \\
\hline 4 & Measurements from both sides & 11 & $\begin{array}{l}\text { Side A } \\
208(73)\end{array}$ & $\begin{array}{l}\text { Side B } \\
216(80)\end{array}$ & \\
\hline
\end{tabular}

Measurements of mucus thickness were made in the four sets of validation experiments. Means (standard deviations) $\mu \mathrm{m}$. (1) with initial measurements and after 30 and 60 minutes; (2) with measurements from adjacent mucosal sections cut of $1.0,1.6$, and $2.6 \mathrm{~mm}$ in thickness; (3) from three adjacent sections of $1.6 \mathrm{~mm}$ thickness; (4) with values from both sides of the section. Comparisons of thickness in each experiment show no statistical difference with the paired $t$ test.

\section{STATISTICAL METHODS}

Mucus thickness for each subject of each site was characterised by the mean of all replicate measurements available. Interobserver variation was qualified by mean (SD) of paired differences, with a paired $t$ test to assess any consistent difference, and a correlation coefficient to show the degree of agreement relative to between subjects variation. The effect of bowel preparation was examined by unpaired $t$ test.

Because there were no elective total colectomies in the control group to permit comparison of mucus thickness at different sites in the same individual, variation between sites was assessed by one way analysis of variance (ANOVA). For the ulcerative colitis and Crohn's disease group, non-orthogonal two way ANOVA was used, many subjects contributing more than one site.

Variation between diagnostic groups at each site was assessed by one way ANOVA, with unpaired $t$ tests for selected contrasts. The correlation of mucus thickness with age was 
TABLE II Mucus thickness in controls and inflammatory bowel disease

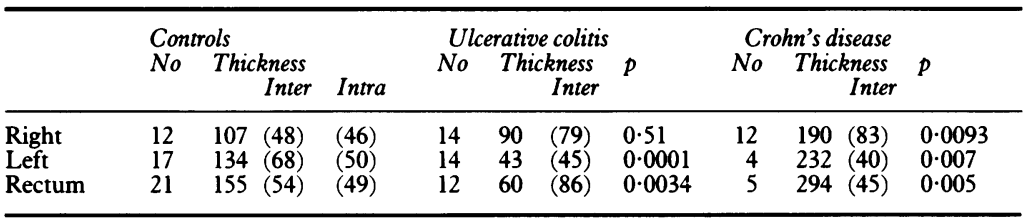

Thickness of adherent mucus, mean (SD) in $\mu \mathrm{m}$ in 50 control specimens from 46 patients, 17 patients which differs from the number of patients. $p$ Values are given for comparisons with controls using which differs from the number of patients. p Values are given for comparisons with controls using unpaired $t$ test. The intersubject SD displays variation between different subjects recorded mean
thickness. The intrasubject SD represents variability of mucus thickness between different samples from the same subject. with ulcerative colitis, and 15 with Crohn's disease. No refers to the number specimens from each site,

bowel obstruction; these were technically unsatisfactory because of gross faecal contamination, ischaemia, and stasis. These data were excluded.

For each anatomical site in the large bowel there was considerable variation in both subject means and variance within subjects. Means for right and left colon and rectum were 107, 134, and $155 \mu \mathrm{m}$ respectively (Fig 2, Table II). The overall $F$ test comparing the three sites did not reach significance $(F=2 \cdot 58 ; \mathrm{p}>0 \cdot 1)$, although the layer in the rectum was significantly thicker than in the right colon $(t=2.61 ; p=0.015$, Table II).

examined for each group and site separately. Analysis of covariance was used to determine the effect of adjusting these contrasts to take account of the considerable age difference between groups.

\section{Results}

Eighty two colectomy specimens were examined from 78 patients of whom four had a second operation with further resection. Fifty specimens were from 46 'control' patients, 17 from patients with ulcerative colitis, and 15 from Crohn's disease patients: During the period these specimens were collected, 19 had surgery for ulcerative colitis, 16 for Crohn's disease, and a number considerably in excess of 46 'control' resections.

\section{MUCUS THICKNESS IN CONTROLS}

An attempt was made to measure thickness in at least two samples from each specimen. In 16 specimens three samples were taken, in 12 two samples, in nine four samples, and in three five samples were taken; in only 10 was a single sample taken.

A continuous layer of mucus could be seen adherent to the epithelium as a distinct layer that could be measured (Fig 1). Exceptions to this were specimens resected for large

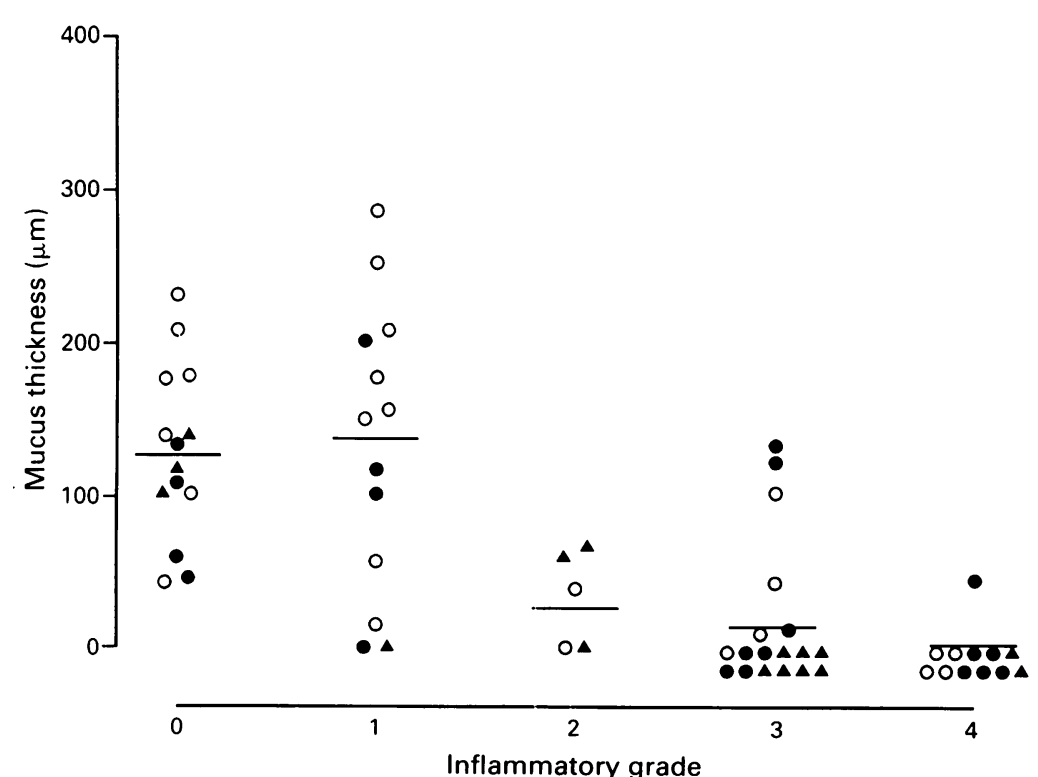

Figure 4: Relation in ulcerative colitis of mucus thickness and inflammatory activity in biopsy specimens from right colon $\bigcirc$, left colon $\mathrm{O}$, and rectum $\Delta$. Inflammation graded 0 and 1 did not differ for mucus thickness although this was significantly reduced for higher grades of inflammation.

\section{MUCUS THICKNESS IN ULCERATIVE COLITIS}

In specimens from the 17 patients with ulcerative colitis we attempted to take two or more samples from each region in the colon. Mucus thickness at the different sites in colitis was more variable compared with controls (Fig 2) because some areas in colitis were denuded of mucus altogether. Analysis of variance shows significant differences between diagnosis groups at each site. Comparisons using the unpaired $t$ test shows no significant difference between controls and colitis on the right side, but a significant difference on the left side and in the rectum (Table II).

To relate the thickness of mucus gel to inflammatory activity in colitis we used standard criteria for inflammatory activity in sections based on Truelove and Richards ${ }^{16}$; this was done without knowledge of values for mucus thickness (Fig 4). In the presence of little or no inflammation (grades $\mathbf{0}$ and $\mathbf{1}$ ) mucus thickness varied greatly with mean values similar to controls. In the presence of more severe inflammatory change (grade 2,3 , and 4) most sections showed little or no mucus adherent to the surface.

\section{MUCUS THICKNESS IN CROHN'S DISEASE}

In specimens from 15 patients we again attempted to obtain at least two samples from each region/specimen. The adherent mucus thickness in Crohn's disease was greater than controls at each site, although only nine findings were on left colon or rectum (Fig 2 and Table II).

Most of the specimens were caecal and related to ileocaecal resections for ileal disease. On the right side seven of 12 specimens from the caecum were macroscopically and microscopically normal but mucus thickness even in these was greater than controls - mean (SD) of the seven was 158 (79) $\mu \mathrm{m}$ compared with controls of 107 (48) $\mu \mathrm{m} \quad(\mathrm{t}=2.61 ; p=0.014)$. Histological inflammatory activity was graded in the same way as sections from ulcerative colitis. Only five of the 15 specimens showed any acute inflammatory change, which in only one was graded severe (grade 4). It was therefore not possible to relate inflammatory activity to mucus thickness in this group.

There was no obvious difference in the gel heterogenicity of the mucus layer in 'controls', ulcerative colitis, and Crohn's disease. 
RELATION OF MUCUS THICKNESS TO AGE AND DRUG TREATMENT

The relation of age and drug treatment at the time of surgery to mucus thickness was examined in both ulcerative colitis and Crohn's disease. Mean ages of patients with ulcerative colitis and Crohn's disease were lower than controls, 47 years (range 22 to 79 ), 38 years (range 18 to 64 ), and 66 years (range 23 to 90 ) respectively but there was no apparent relation between mucus thickness and age. In an additional analysis, we took age into account as a covariate to correct for differences in ages of the groups. Qualitatively the pattern of data is not substantially changed, although the differences between Crohn's disease and controls for the left colon failed to achieve statistical significance.

In colitis 12 of 17 were taking steroids in combination with 5-ASA in nine of them. Two were receiving no treatment at all and three receiving 5-ASA alone. Patients with less severe disease were those not taking steroids. Reduction in mucus thickness, however, seemed to bear little relation to drugs given in colitis. There was an overriding effect from active inflammation at the site where measurements were made. In five patients receiving no treatment or 5-ASA alone, measurements from more than one site in four of them showed low values at sites of acute inflammation and normal values where there was little or no acute change.

In Crohn's disease, nine patients were taking steroids combined with 5-ASA in seven, three received no treatment and three 5-ASA alone. Values were similar in those with and without steroids.

\section{Discussion}

Our findings identify, for the first time, that there is a continuous layer of adherent mucus gel coating the human colon. In patients with ulcerative colitis the absence of any surface mucus on severely inflamed mucosa was in striking contrast with the thick layer seen in Crohn's disease.

The absence of an adherent mucus layer in conventional preparations of tissue for histological examination is caused by dehydration during fixation, which leads to destruction of the complex mucus gel structure; only traces of surface mucus remain on mucosa in such sections. One must question whether the layer we have studied in fresh sections is indeed mucus. It reacts readily with both PAS and Alcian blue, which stain neutral and acidic mucus respectively. Furthermore the technique we have used has previously been validated for measurements of both gastric and colonic mucus in animals with some findings on the stomach in humans. ${ }^{7}$ The series of experiments we describe to validate the method show that it is both reliable and reproducible; the mucus gel remains stable for at least 60 minutes, may be turned over on the slide, and is unaffected by the thickness of cut sections (Table I). There was also a good correlation in measurements by two independent observers.

All the surgical specimens were examined within 20 minutes of resection with minimal handling of specimens. 'Control' data were largely derived from patients with carcinoma of the colon and may not represent 'normal' findings; information from other 'control' patient groups, perhaps obtained by biopsy would be of interest in the future. Multiple samples were taken from specimens of inflammatory bowel disease to include different anatomical sites and areas of inflammation as well as comparatively 'normal' mucosa. Obstructed bowel removed during emergency surgery was unsuitable for our study because the surface was heavily contaminated, which made the mucus layer difficult to identify. Thickness of the mucus layer even in the control group varied between 48 to $273 \mu \mathrm{m}$ depending partly on the site. There was considerable intrasubject and intersubject variability. The circumstances for all patients before surgery were very similar and included standard bowel preparation with oral fluids only for the previous 24 hours.

It is not possible to compare our findings with other studies because there have been no measurements of colonic mucus thickness in humans. It is also unlikely that bias would affect our results, as we were not in a position to anticipate any of the values from any of the groups. There have, however, been attempts to visualise the mucus layer by other techniques. The 'pachymeter', which entails a slit lamp and image splitting system has been used to obtain measurements of mucus from fresh rat colon. ${ }^{17}$ Attempts to preserve mucus before fixation include stabilisation with antimucin antisera ${ }^{18} 19$ or celloidin on frozen sections ${ }^{2021}$ and cryostat sectioning plus freeze substitution/vapour fixation. ${ }^{22}$ None of these methods has been used on human colon and measurements of thickness have varied greatly depending on the technique used.

Thickness of the mucus layer is related to the dynamic equilibrium between mucus secretion and its subsequent removal into the lumen. In ulcerative colitis the absence of an adherent layer in some areas may be a result of inadequate secretion or excessive removal. As the epithelial layer was maintained in specimens graded 1 to 3 , the absence of mucus could not be accounted for on the basis of simple exfoliation. One cannot conclude very much from goblet cell depletion of mucus in this condition, which has been attributed to immaturity of an epithelium turning over rapidly. ${ }^{122324}$ Various studies on mucus synthesis in colitis would suggest that provided the epithelial layer remains intact in the presence of mild to moderate inflammation, mucus synthesis is either normal or increased. In the presence of severe inflammation or inactivity, however, it tends to be lower than normal. ${ }^{25-28}$

Abnormalities in the mucus of ulcerative colitis have been shown by histochemical studies of the constituent mucus glycoproteins, which show changes in the degree of sialation and sulphation $^{29-31}$ with shortened oligosaccharide side chains $^{1011}$ and changed lectin binding. ${ }^{32} 33$ Podolsky has found a depletion of one specific mucin subspecies proved both by anion exchange chromatography and monoclonal antimucin antibodies. ${ }^{34-36}$ It seems that failure of intracellular storage of this mucin species may be 
responsible $^{37}$ though others have doubted the significance..$^{38}$ The implications of these findings in colitis are not clear, but they may well influence the gel forming properties of mucus; the gel may be less viscous and form a less competent barrier against luminal agents with the capacity to breakdown mucus and damage mucosa. ${ }^{13} 14$

The apparent increase in thickness of mucus in Crohn's disease requires further investigation, particularly as our initial findings include few specimens from the left colon and rectum. It is perhaps another feature distinguishing ulcerative colitis from Crohn's disease, for the inflammatory changes in colitis are diffuse and limited to mucosa, compared with patchy inflammation in Crohn's disease, which affects the whole bowel wall. The patchy involvement of mucosa in Crohn's disease may be responsible through various inflammatory mediators for excessive mucus secretion, which is not immediately shed into the lumen because the epithelium is largely intact on intervening areas of comparatively normal mucosa with intact epithelium. Goblet cell numbers and mucin staining characteristics are normal in Crohn's disease. Dvorak et $a l^{39}$ used scanning electron microscopy to examine the terminal ileum of Crohn's disease patients and found an abnormally thick layer of mucus with engorged goblet cells. Unfortunately these findings were not extended to the colon; neither have we looked at the small bowel for Crohn's disease. From animal work is is known that the mucus layer in small bowel is discontinuous, in contrast with that in the colon. ${ }^{18}$

The adherent layer of mucus gel on colonic mucosa is probably important in normal health because it is the interface between luminal contents and epithelium. The thick layer of mucus is maintained by an equilibrium with freshly secreted mucus replacing that which is lost by mechanical shear of faeces and digestion by luminal proteinase enzyme..$^{41}$ Faecal proteinases that are largely bacterial in origin are present in ulcerative colitis in concentrations three times greater than normal. ${ }^{42}$ Protein exuding through the surface mucus in ulcerative colitis may enhance growth of proteinase producing organisms by providing their substrate. The enzymes are also derived from white cells, which would be shed into the lumen. It is possible that a change in colonic bacteria, or in their ability to produce proteinase may play some part in the pathogenesis of colitis by removing surface mucus at a greater rate than normal. It cannot account, however, for the whole picture because the mucus layer seemed normal in areas that were not inflamed. Changes in other enzyme concentrations have also been seen in faeces of colitis patients and include raised concentrations of sulphatase, ${ }^{43}$ glycosidases, and a novel 0 acetylsialic acid esterase. ${ }^{40}$ Colonic contents in colitis may break down mucus more readily because of raised luminal proteinases with the additional effect of glycosidases, sulphatases, and esterases all of which would render both mucus and mucosa more liable to damage. On the other hand it could be argued that the changes in bacterial enzymes are largely secondary phenomena. Changes in mucosal prosta- glandins or other eicosanoids may play an important part in maintaining the surface mucus gel..$^{44}$

Our findings warrant further investigation to explore the role of colonic mucus in inflammatory bowel disease, because agents that maintain a healthy layer of mucus gel on colonic mucosa could be of therapeutic value in ulcerative colitis.

We are grateful to surgical colleagues for providing fresh colonic We are grateful to surgical colleagues for providing fresh colonic tissue. Professor L E Hughes provided facilities in the University Department of Surgery and Mr Glan Morgan in the Department of Medical Bioengineering made the cutting device. Illustrations
were provided by the Department of Medical Illustration, and $\mathrm{Mr}$ were provided by the Department of Medical Illustration, and $\mathrm{Mr}$
Dilwyn Havard, Department of Histopathology, produced the cryostat frozen sections.

1 Smith AC, Podolsky DK. Colonic mucin glycoproteins in health and disease. Clinics in Gastroenterology 1986; 15: 81537.

2 Neutra MR, Forstner JF. Gastrointestinal mucus; synthesis, secretion function. In: Johnson LR, ed. Physiology of the GI tract. 4th ed. vol 2. New York: Raven Press, 1987: 9751009.

3 Allen A, Hoskins L. In: Kirsner JB, Shorter RG, eds. Disease of the colon and rectum. Baltimore, Maryland: Williams \& Wilkins, 1988: 65-92.

4 Allen A. Gastrointestinal mucus. In: Forte JG, ed. Handbook of physiology: the gastrointestinal system. vol III. Washington, DC: American Physiological Society, 1989: 359-82.

5 Florey HW. Observations on the functions of mucus and the early stages of acterial invasion of the intestinal mucosa. early stages of acterial invasion of the intestinal m
fournal of Pathology and Bacteriology 1933; 37: 283-9.

6 Florey $\mathrm{H}$. Mucin and the protection of the body. Proc $R$ Soc Lond 1955; 143: 144-8.

7 Kerss S, Allen A, Gardner A. A simple method for measuring thickness of the mucosa gel layer adherent to rat, frog and human gastric mucosa: influence of feeding, prostaglandin $\mathrm{N}$-acetyl and other agents. Clin Sci 1982; 63: 187-95.

8 Allen A, Bell A, McQueen S. Mucus and mucosal protection. In: Allen A, ed. Mechanisms of mucosal protection in the upper GI tract. New York: Raven, 1984: 195-201.

9 Soergel KH, Ingelfinger FJ. Composition of rectal mucus in normal subjects and patients with ulcerative colitis. Gastroenterology 1964; 47: 610-6.

10 Teague RM, Fraser D, Clamp JR. Changes in monosaccharide content of mucus glycoproteins in ulcerative colitis. $B M \mathcal{F}$ 1973; 2: 645-6.

11 Clamp JR, Fraser G, Read AE. Studies of the carbohydrate content of mucus glycoproteins from normal and diseased content of mucus glycoproteins $\mathrm{f}$
colons. Clin Sci 1981; 61: 229-34.

12 McCormick DA, Horton LWL, Mee AS. Mucin depletion in inflammatory bowel disease. $\mathcal{F}$ Clin Pathol 1990; 43: 143-6.

13 Rhodes JM. Colonic mucus and mucosal glycoproteins: the key to colitis and cancer? Gut 1989; 30: 1660-6.

14 Allen A, Hutton DA, Pearson JP, Sellers LA. The colonic mucus gel barrier: structure, gel formation and degradation. In: Peters TJ, ed. The cell biology of inflammation in the gastrointestinal tract. Hull: Corners Publications, 1990: 11325 .

15 Lennard-Jones JE. Classification of inflammatory bowel disease. Scand $\mathcal{F}$ Gastroenterol 1989; 24: (suppl 170): 2-6.

16 Truelove SC, Richards WCD. Biopsy studies in ulcerative colitis. BMF 1956; 2: 1315-21.

17 Bickel M, Kauffman GL. Gastric gel mucus and effect of distension, 16,16-dimethyl PGE2. Gastroenterology 1981; 80: $770-5$

18 Rozee KR, Cooper D, Lam K, Costerton JW. Microbial flora of the mouse ileum, mucus layer and epithelial surface. Appl Environ Microbiol 1982; 43: 1451-63.

19 Bollard JE, Vanderwee MA, Smith GW, Tasman-Jones C, Gavin JB, Lee S. Preservation of mucus in situ in rat colon. Dig Dis Sci 1986; 31: 1338-44.

20 Szentkuti L, Eggers A. Stabilisation of pre-epithelial mucus gel in cryostat sections from rat colon and celloidin. Stain Technol 1990; 65: 179-81.

21 Szentkuti L, Riedesel H, Enss ML, Gaertner K, Von Engelhardt W. Pre-epithelial mucus layer in the colon on conventional and germ-free rats. Histochem $\mathcal{F} 1990 ; 22$ : conven $491-7$.

22 Sagata T, Engelhard WV. Luminal mucin in the large intestine of mice, rats and guinea pigs. Cell Tissue Res 1981; 219: 62935 .

23 Filipe MI. Mucins in the human GI epithelium: a review. Invest Cell Pathol 1979; 2: 195-216.

24 Morson BC, Dawson IMP, Day DW, Jass JR, Prince AB, Williams GT. Morson E' Dawson's Gastointestinal pathology. 3rd ed. Oxford: Blackwell Scientific Publications, 1990.

25 MacDermott RP, Donaldson RM, Trier JS. Glycoprotein synthesis and secretion by mucosal biopsies of rabbit colon and rectum. $\mathcal{F}$ Clin Invest 1974; 54: 545-54.

$26 \mathrm{Kim}$ YS, Isaacs R. Glycoprotein metabolism in inflammatory and neoplastic diseases of the human colon. Cancer Res 1975 ; 35: 2092-7.

27 Goodman MJ, Kent PW, Truelove SC. Glucosamine synthetase activity of the colonic mucosa in ulcerative colitis and Crohn's disease. Gut 1977; 18: 219-28.

28 Cope GF, Heatley RV, Kelleher J, Axon ATR. In vitro mucus glycoprotein production by colonic tissue from patients with ulcerative colitis. Gut 1988; 29: 229-34. 
29 Filipe MI, Dawson I. The diagnostic value of mucosubstances in rectal biopsies from patients with ulcerative colitis and Crohn's disease. Gut 1970; 11: 229-34.

30 Culling CFA, Reid PE, Dunn WI. A histochemical comparison of the $\mathbf{0}$-acylated siatic acids of the epithelial mucins in ulcerative colitis, Crohn's disease and normal controls. $\mathcal{f}$ Clin Pathol 1979; 32: 1272-7.

31 Habib NA, Dawson PM, Krausz T, Blount MA, Kersten D Wood CB. A study of histochemical changes in patients with ulcerative colitis, Crohn's disease and DD of colon. Dis ulcerative colitis, Crohn's dise

32 Jacobs LR, Huber PW. Regional distribution and alternations of lectin binding to colorectal mucin in mucosal biopsie from controls and subjects with inflammatory bowel disease. f Clin Invest 1985; 75: 112-8.

33 Rhodes JM, Black RR, Savage A. Altered lectin binding by colonic epithelia glycoconjugates in ulcerative colitis and Crohn's disease. Dig Dis Sci 1988; 33: 1359-63.

34 Podolsky DK, Isselbacher KJ. Composition of human colonic mucin: alterations in inflammatory bowel disease. $\mathcal{F}$ Clin Invest 1983; 72: 142-53.

35 Podolsky DK, Isselbacher KR. Glycoprotein composition of colonic mucosa. Specific alterations in ulcerative colitis. colonic mucosa. Specific alteration
Gastroenterology 1984; 87: 991-8.

36 Podolsky DK, Fournier DA. Alterations in mucosal content of colonic glycoconjugates in inflammatory bowel disease defined by monoclonal antibodies. Gastroenterology 1988; 95: 379-87.

37 Smith AC, Podolsky DK. Biosynthesis and secretion of human colonic glycoproteins. $\mathcal{F}$ Clin Invest 1987; 80: 300-7.

38 Rhodes JM, Parker N, Ching CK, Patel P. Mucin subclasses in ulcerative colitis (letter and reply). Gastroenterology 1987; 93: $435-6$.

39 Dvorak AM, Connell AB, Dickersin GR. Crohn's disease: a scanning electron microscopic study. Hum Pathol 1979; 10: 165-77.

40 Corfield AP, Williams AJF, Clamp JR, Wagner SA, Mountford RA. Degradation by bacterial enzymes of colonic mucus rom normal subjects and patients with inflammatory bowel disease: the role of sialic acid metabolism and the detection f a novel 0 -acetylsialic acid esterase. Clin Sci 1988; 74: 71-8

41 Hutton DA, Pearson JP, Allen A, Foster SNE. Mucolysis of he colonic mucus barrier by faecal proteinases; inhibition by interacting polyacrylate. Clin Sci 1990; 78: 265-71.

42 Samson HJ, Allen A, Pearson JP, Cunliffe WJ, Rhodes M, Rhodes J. Faecal proteinase activity; raised value in patients with ulcerative colitis. Gut 1991; 32: A1235-A6.

43 Rhodes JM, Gallimore R, Elias E, Kennedy JF. Faeca sulphatase in health and in inflammatory bowel disease. Gut 1985; 26: 466-9.

44 Zijlstra FJ, Srivastava ED, Rhodes M, van Dijk APM, Fogg F, Samson HJ, et al. Effect of nicotine on rectal mucus and mucosal eicosanoids. Gut (in press). 